\title{
Liver acid sphingomyelinase inhibits growth of metastatic colon cancer
}

\author{
Yosuke Osawa, ${ }^{1,2,3}$ Atsushi Suetsugu, ${ }^{2}$ Rie Matsushima-Nishiwaki, ${ }^{1}$ Ichiro Yasuda, ${ }^{2}$ \\ Toshiji Saibara, ${ }^{4}$ Hisataka Moriwaki, ${ }^{2}$ Mitsuru Seishima, ${ }^{3}$ and Osamu Kozawa ${ }^{1}$
}

1Department of Pharmacology, 2Department of Gastroenterology, and 3Department of Informative Clinical Medicine, Gifu University Graduate School of Medicine, Gifu, Japan. ${ }^{4}$ Department of Gastroenterology and Hepatology, Kochi University School of Medicine, Kochi, Japan.

\begin{abstract}
Acid sphingomyelinase (ASM) regulates the homeostasis of sphingolipids, including ceramides and sphingosine-1-phosphate (S1P). These sphingolipids regulate carcinogenesis and proliferation, survival, and apoptosis of cancer cells. However, the role of ASM in host defense against liver metastasis remains unclear. In this study, the involvement of ASM in liver metastasis of colon cancer was examined using $\mathrm{Asm}^{-/-}$and $\mathrm{Asm}^{+/+}$mice that were inoculated with SL4 colon cancer cells to produce metastatic liver tumors. Asm ${ }^{-/-}$mice demonstrated enhanced tumor growth and reduced macrophage accumulation in the tumor, accompanied by decreased numbers of hepatic myofibroblasts (hMFs), which express tissue inhibitor of metalloproteinase 1 (TIMP1), around the tumor margin. Tumor growth was increased by macrophage depletion or by Timp1 deficiency, but was decreased by hepatocyte-specific ASM overexpression, which was associated with increased S1P production. S1P stimulated macrophage migration and TIMP1 expression in hMFs in vitro. These findings indicate that ASM in the liver inhibits tumor growth through cytotoxic macrophage accumulation and TIMP1 production by hMFs in response to S1P. Targeting ASM may represent a new therapeutic strategy for treating liver metastasis of colon cancer.
\end{abstract}

\section{Introduction}

Colon cancer, one of the most common malignancies, frequently metastasizes to the liver. Acid sphingomyelinase (ASM) is involved in various physiological cellular functions and diseases, including cancer (1), and hydrolyzes sphingomyelin into ceramide and phosphorylcholine. Ceramide, a bioactive mediator of numerous cellular functions, such as apoptosis and cell cycle regulation $(2,3)$, is in turn hydrolyzed by ceramidase into sphingosine, which is subsequently phosphorylated into sphingosine-1-phosphate (S1P) by sphingosine kinase (SphK). Although these sphingolipids are involved in colon carcinogenesis and colon cancer cell survival $(4,5)$, the roles of ASM and S1P in host antitumor defenses (i.e., inhibiting the progression of colon cancer metastasis to the liver) remain unclear.

Tumors contain stromal cells, such as immune cells and fibroblasts (6). Infiltrated tumor-associated macrophages (TAMs) have recently been reported to function as promoters of tumor progression (7-9), with several clinical studies demonstrating an association between the presence of TAMs and poor prognosis in various cancers (10). In contrast, opposing data have shown that the presence of TAMs is correlated with improved survival and that these cells have protective potential in colon cancer $(11,12)$. Although tumor growth-promoting TAMs have been observed to resemble regulatory M2 macrophages $(13,14)$, the density of classically activated M1 macrophages is positively correlated with the survival time of patients with non-small-cell lung cancer (15). These findings indicate that macrophages have contrasting roles in cancer, depending on their phenotype (13).

$\mathrm{S} 1 \mathrm{P}$ is a ceramide-derived metabolite that is involved in various cellular functions and increases migration of macrophages $(16,17)$, resulting in macrophage recruitment. S1P released from apoptotic tumor cells induces a switch from the M1 to the M2

Conflict of interest: The authors have declared that no conflict of interest exists. Citation for this article: J Clin Invest. 2013;123(2):834-843. doi:10.1172/JCI65188. macrophage subtype (18). In contrast, the S1P analog FTY720 does not alter the ratio of M1 and M2 subtypes in mouse peritoneal macrophages (19). Because of these contrasting findings, the effect of S1P on macrophages remains controversial and poorly understood. Whereas MMP cleavage of the ECM, a primary barrier against tumor invasion produced by hepatic myofibroblasts (hMFs) in the liver, promotes cancer cell migration, invasion, and metastasis, MMP inhibitors reduce colon cancer metastasis to the liver (20). Specifically, overexpression of tissue inhibitors of metalloproteinases (TIMPs), endogenous inhibitors of MMPs, inhibits liver metastasis of colon cancer in animal models (21), whereas decreased TIMP1 expression in the liver results in progression of SV40T antigen-induced hepatocellular carcinoma (22) and increased metastatic colonization of T cell lymphoma (23). However, it has been reported that elevated TIMP levels are associated with cancer progression (24). Thus, although S1P stimulates hMF accumulation and TIMP1 induction in the liver $(25,26)$, the precise roles of S1P in tumor growth remain unclear. To attempt to clarify these precise roles, we here investigated the involvement of ASM in the progression of liver metastasis of colon cancer.

\section{Results}

Role of ASM deficiency and overexpression in the progression of metastatic liver tumors of colon cancer. To determine the role of host ASM on metastatic liver tumor growth caused by colon cancer, we created metastatic liver tumors by intrasplenic injection with SL4 cells, a mouse colon cancer cell line, and examined expression of ASM and ceramide by immunostaining. ASM and ceramide expression increased in the liver cells around the tumors (Supplemental Figure 1A; supplemental material available online with this article; doi:10.1172/JCI65188DS1), which suggests that tumor cells stimulate ASM expression and promote its activity in liver cells. To examine the effect of ASM deficiency on metastatic liver tumors, the extent of tumor growth in $\mathrm{Asm}^{+/+}$and $\mathrm{Asm}^{-/-}$mice 
A
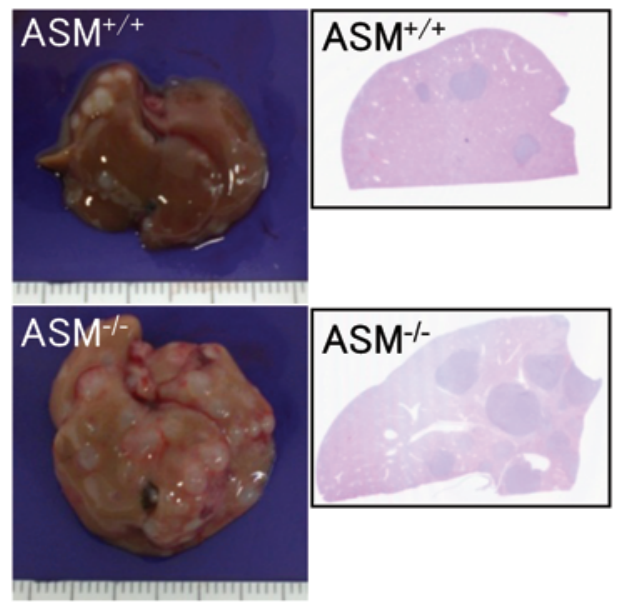

B
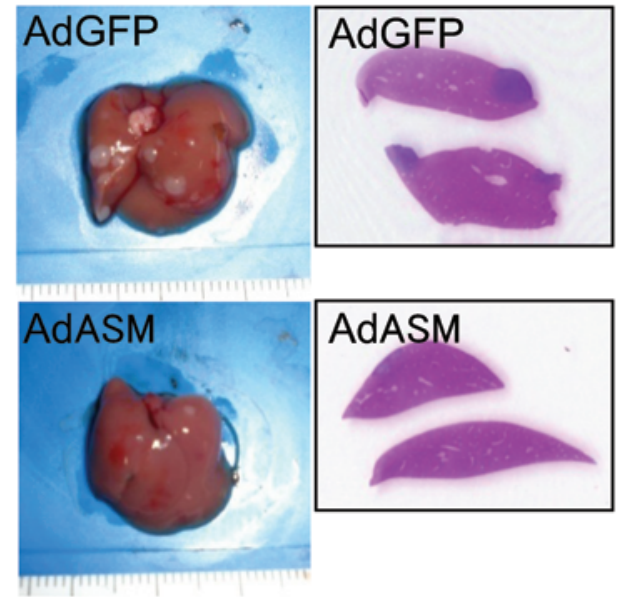

C
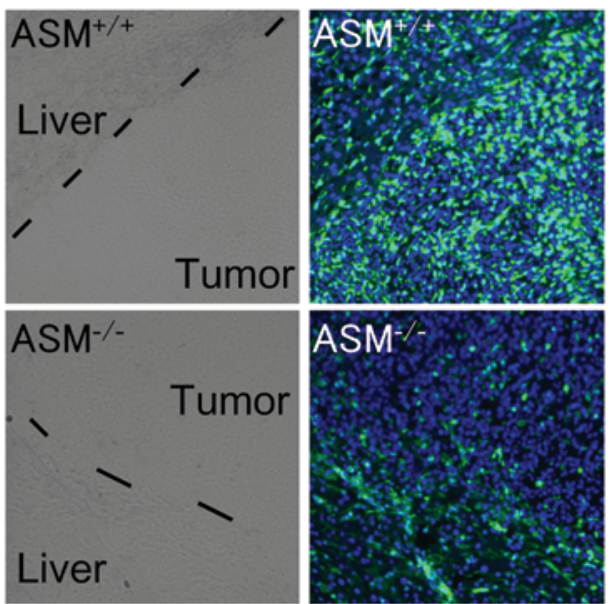
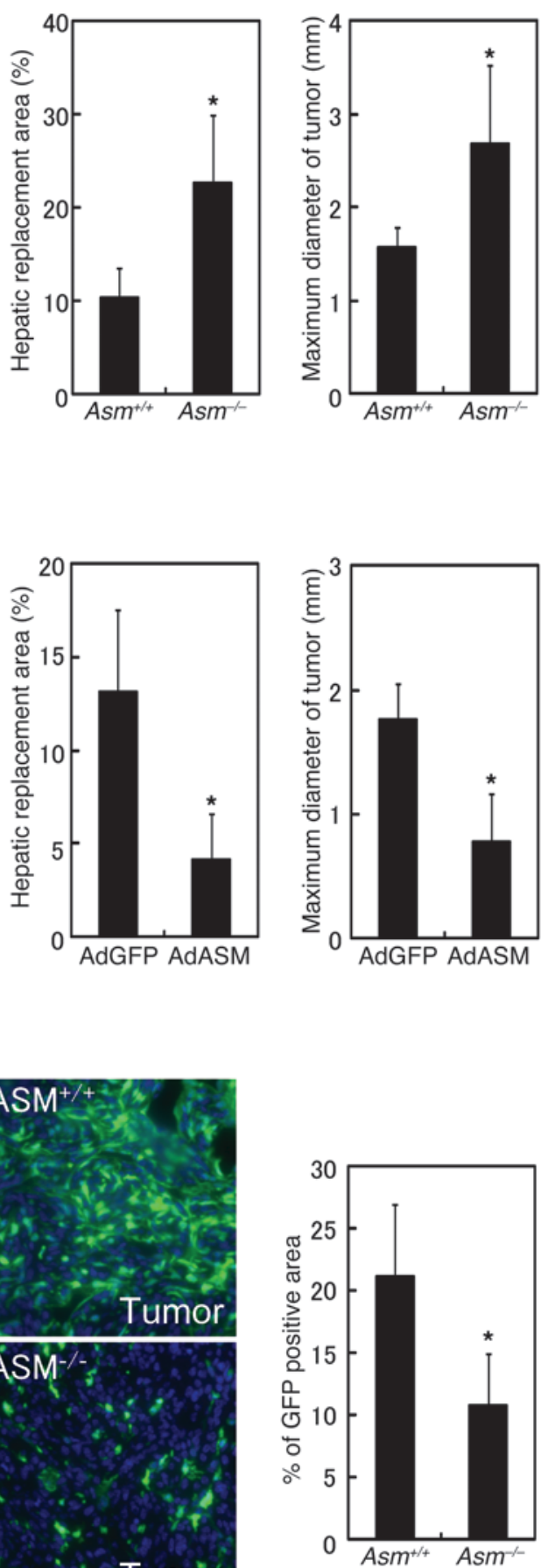

Figure 1

ASM deficiency increased, and ASM overexpression decreased, metastatic tumor growth in the liver. (A and $\mathbf{B}$ ) $\mathrm{Asm}^{+/+}$and $\mathrm{Asm}^{-/-}$mice were intrasplenically injected with $2 \times 10^{4}$ SL4 cells (A). Wildtype mice were infected with AdGFP or AdASM 24 hours before inoculation (B). Mice were sacrificed 14 days after inoculation, and livers were excised and photographed. Liver sections were stained with H\&E (loupe magnification), and intrahepatic tumor load was presented as hepatic replacement area and maximum diameter, based on measurement of 3 nonsequential sections. (C) $\mathrm{GFP}^{+} \mathrm{Asm}^{+/+}$and $\mathrm{GFP}^{+} \mathrm{Asm}^{-/-}$mice were intrasplenically injected with SL4 cells. At 14 days after inoculation, GFP expression in the liver was assessed by fluorescent microscopy, measurement of the $\mathrm{GFP}^{+}$area was performed, and nuclei were stained with DAPI. Shown are bright field images at the border of the metastatic tumor and liver (left), GFP/DAPI merge in the same bright field images (middle), and highermagnification views of GFP/ DAPI merge in the tumor area (right). Original magnification, $\times 200$ (left and middle); $\times 400$ (right). Results are mean \pm SD of data obtained from at least 5 independent experiments. ${ }^{*} P<0.05$, 2-tailed Student's $t$ test. was compared. No tumors were evident in livers without SL4 cell inoculation (Supplemental Figure 1B). After inoculation with SL4 cells, the hepatic replacement area, maximum tumor diameter, and liver weight were increased in $\mathrm{Asm}^{-/-}$compared with $\mathrm{Asm}^{+/+}$mice (Figure 1A and Supplemental Figure 1C). In contrast, a similar number of the initially transplanted cells was observed in the livers of $\mathrm{Asm}^{+/+}$and $\mathrm{Asm} \mathrm{sm}^{-/-}$mice 6 hours after inoculation (Supplemental Figure 2A). These results indicate that colon cancer cells grow more rapidly in the $A s m^{-/}$liver. Conversely, administration of adenovirus-expressing ASM (AdASM) to the mice prior to cancer cell inoculation inhibited tumor growth compared with adenovirus-expressing GFP (AdGFP) administration (Figure 1B). AdASM-derived GFP was expressed in the hepatocytes, but not in the tumor cells (Supplemental Figure 2B). In support of the results of our previous study (27), AdASM increased ASM activity in the liver (Supplemental Figure 2C). In addition, ASM-chimeric mice, 
in which ASM was deficient only in bone marrow-derived cells, demonstrated tumor growth that was comparable to mice containing $\mathrm{Asm}^{+/+}$bone marrow cells (Supplemental Figure 3A). These results indicate that ASM in hepatocytes, but not in bone marrow-derived cells, is involved in the inhibition of tumor growth.

To explore the mechanisms of enhanced tumor growth in $\mathrm{Asm}^{-/}$ mice, we generated $G F P^{+} A s m^{-/-}$mice and examined the host-tumor interaction by inoculating $\mathrm{GFP}^{+} \mathrm{Asm}^{+/+}$and $\mathrm{GFP}^{+} \mathrm{Asm} \mathrm{s}^{-/-}$mice with SL4 cells. After inoculation, spindle-shaped host-derived $\mathrm{GFP}^{+}$cells, most of which expressed F4/80 (Figure 2A), were observed in the metastatic liver tumors, while fewer host-derived cell infiltrates were observed in the $\mathrm{Asm}^{-/}$livers (Figure 1C). Spindle-shaped bone marrow-derived cells were also observed in the tumors of GFPchimeric mice (expressing GFP only in bone marrow-derived cells; Supplemental Figure 3B), which suggests that these cells were $\mathrm{F} 4 / 80^{+}$macrophages originating from the bone marrow. F4/80 cells were observed in the tumors; compared with the control livers, a smaller number of F4/80 $0^{+}$cells was observed in the $\mathrm{Asm}^{-/-}$livers, while a greater number of these cells was present in AdASM-infected livers (Figure 2, B and C). The induction of classically activated M1 macrophage markers (Cd11c, Il12p40, and Ifng) by SL4 cell inoculation was more remarkable than that of $C d 163$, mannose receptor, and Il10, markers for regulatory M2 macrophages (Supplemental Table 1). Thus, these findings suggested that the accumulated macrophages were M1 dominant. In $\mathrm{Asm}^{+/+}$mice transplanted with $\mathrm{GFP}^{+} \mathrm{Asm}^{-/-}$bone marrow, the number of bone marrow-derived $\mathrm{GFP}^{+} \mathrm{Asm}^{-/-}$cells in the tumor was comparable to that of $\mathrm{GFP}^{+} \mathrm{Asm} \mathrm{sm}^{+/+}$ cells in the control $\mathrm{Asm}^{+/+}$mice transplanted with $\mathrm{GFP}^{+} \mathrm{Asm}^{+/+}$bone marrow (Supplemental Figure 3C). Therefore, it is likely that ASM in hepatocytes, but not in bone marrow-derived cells, contributes to the accumulation of macrophages in tumors.

To examine whether macrophage accumulation in tumors is involved in the inhibition of tumor growth, liposome-encapsulated alendronate was administered to the SL4 cell-inoculated mice to decrease the number of F $4 / 80^{+}$cells in the tumors (Supplemental Figure 3D). Alendronate administration increased the severity of tumor growth by inducing macrophage depletion (Figure 2D), which is suggestive of macrophage involvement in tumor growth suppression. These data indicate that hepatocytic ASM leads to accumulation of antitumor macrophages in the metastatic liver tumors of colon cancer, thereby suppressing tumor growth.

Macrophage-induced hMFs accumulate around invasive margins of metastatic liver tumors. Macrophages have been shown to exhibit antitumor potential as tumoricidal cells and major antigen-presenting cells (28-31). Liver macrophages also modulate the host immune response to cancer cells by releasing cytotoxic products and immune-stimulating factors, including IFN- $\gamma$. In accordance with these findings, $\mathrm{CD}^{+}$lymphocytes infiltrated into the tumors, and fewer $\mathrm{CD}^{+}$cells were observed in $\mathrm{Asm}^{-/-}$and alendronate-treated mice (Supplemental Figure 4, A and B). Moreover, a NKT cell activator, $\alpha$-galactosylceramide, suppressed tumor growth, even in $\mathrm{Asm}^{-/-}$mice (Supplemental Figure 4C), which suggests that the antitumor immunity of NKT cells was not impaired in $\mathrm{Asm}^{-/-}$mice. Thus, NKT cells contributed to the antitumor defense mechanism.

Activated macrophages release various types of inflammatory cytokines and growth factors. Among these, TNF- $\alpha$ is thought to induce tumor necrosis. However, in the model used in this study, mRNA expression of Tnfa was comparable in SL4 cell-inoculated $\mathrm{Asm}^{-/-}$and $\mathrm{Asm}^{+/+}$livers (Supplemental Table 2), and tumor growth was not increased in $\mathrm{Tnfa}^{-/-}$mice (Supplemental Figure 4D), which suggests that TNF- $\alpha$ is not involved in tumor development in Asm ${ }^{-/-}$mice. The reduced induction of Il1b, Cxcl1, and Tgfb mRNA expression by SL4 cell inoculation in $\mathrm{Asm}^{-/-}$compared with $\mathrm{Asm}^{+/+}$ livers (Supplemental Table 2) can be explained by the reduction in the number of macrophages in the $\mathrm{Asm}^{-/}$liver, as these factors are released from liver macrophages. The mRNA expression levels of Des (encoding desmin, a marker of hMFs), Acta2 (encoding $\alpha$-SMA, a marker of activated hMFs), and Col1a1 (encoding collagen $\alpha 1[\mathrm{I}]$, a product of activated hMFs) were also increased after SL4 cell inoculation in $\mathrm{Asm}^{+/+}$livers (Supplemental Table 2), which suggests that the metastatic tumors stimulated hMF proliferation and activation. In the SL4 cell-inoculated mice, the number of desmin ${ }^{+}$and $\alpha$-SMA ${ }^{+}$ cells were increased around the invasive margins of the tumors in the liver (Figure 3). Desmin ${ }^{+}$cells were observed to express GFP in the $\mathrm{GFP}^{+}$mice, but not in the GFP-chimeric mice (data not shown), which suggests that the hMFs were derived from host, not from bone marrow. The increase of Des, Acta2, and Col1a1 mRNA after SL4 cell inoculation was lower in $\mathrm{Asm}^{-/-}$than in $\mathrm{Asm}^{+/+}$livers (Supplemental Table 2). Moreover, whereas the number of desmin ${ }^{+}$and $\alpha-\mathrm{SMA}^{+}$ cells was decreased by Asm deficiency (Figure 3A) and by macrophage depletion (Figure 3C), it was increased by ASM overexpression (Figure 3B). These findings suggest that increased macrophage accumulation in the metastatic liver tumor by ASM stimulates hMF accumulation and activation. Because the number of desmin ${ }^{+}$and $\alpha-\mathrm{SMA}^{+}$cells was inversely correlated with tumor growth, we concluded that hMFs may contribute to tumor suppression.

Role of TIMP1 produced by hMFs in growth suppression of metastatic liver tumors. Although it has been reported that hMFs contribute to cancer progression, the model used in this study indicated that increased hMF accumulation is negatively correlated with tumor growth. This finding led us to hypothesize that hMFs contain antitumor factors that are increased in number and magnitude by ASM overexpression and decreased by ASM deficiency. To test this hypothesis, we focused on examining TIMP1, which exhibits antitumor potential (21) and is expressed by hMFs (32). In the metastatic tumors, most of the desmin ${ }^{+}$cells showed double staining for TIMP1 (Figure 4A), which suggests that hMFs are TIMP1+. Timp1 mRNA expression increased in $\mathrm{Asm}^{+/+}$livers by SL4 cell inoculation, an increase that was attenuated in Asm ${ }^{-1-}$ livers (Figure 4B). Nevertheless, the expression levels of Mmp2, Mmp9, and Mmp13 were comparable in $\mathrm{Asm}^{+/+}$ and $A \mathrm{sm}^{-/-}$livers (Supplemental Table 2). The increased number of $\mathrm{TIMP}^{+}$cells observed around the invasive margin of the tumors was attenuated by Asm deficiency as well as by macrophage depletion (Figure 4, C and E), whereas ASM overexpression further increased TIMP1 $1^{+}$cell number (Figure 4D), similar to our findings with respect to hMF induction. These results, in addition to the increased tumor growth observed in Timp $1^{-/-}$mice (Figure 4F), suggest that the increased expression of TIMP1 in hMFs by metastatic tumors is involved in the suppression of tumor growth.

ASM suppression of tumorgrowth via S1P production. In accordance with previous reports that ASM hydrolyzes sphingomyelin into ceramide, which is then hydrolyzed by ceramidase and phosphorylated by SphK to form S1P, we observed the level of S1P to increase in AdASM-infected livers (Figure 5A). Although exogenous administration of S1P did not affect Tnfa, Il1b, Cxcl1, Tgfb, Il12p40, or Il10 mRNA expression in isolated peritoneal $\mathrm{CD}_{11 \mathrm{~b}^{+}}$macrophages (Supplemental Table 3), these cells showed increased migration toward S1P (Figure 5B), as previously reported regarding RAW 264.7 macrophages (33). These findings suggest that macrophage accumulation in tumors might be caused by S1P-induced macrophage recruitment. Although it 
A

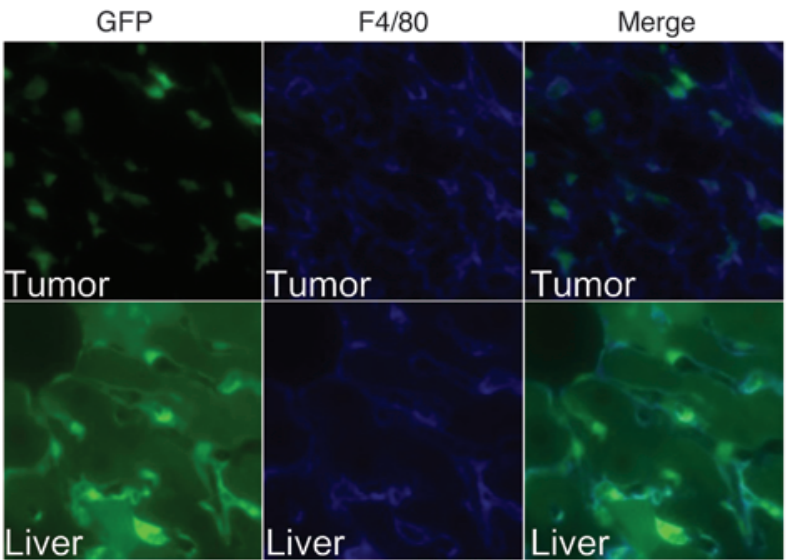

B

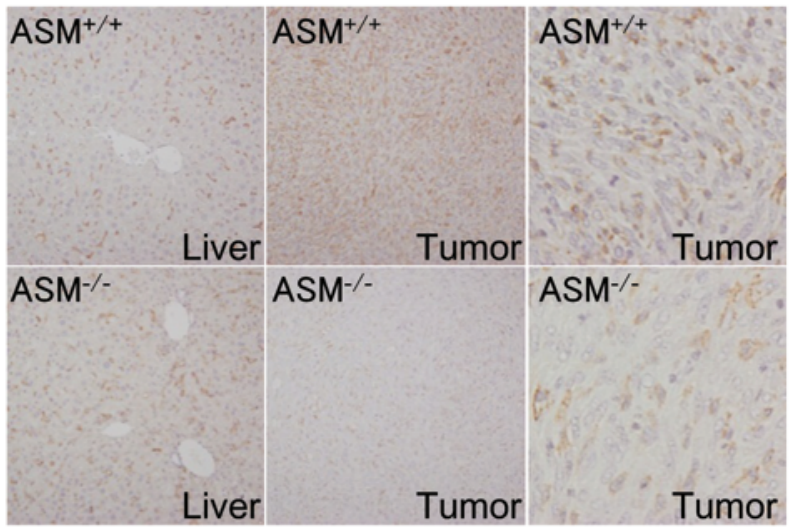

C

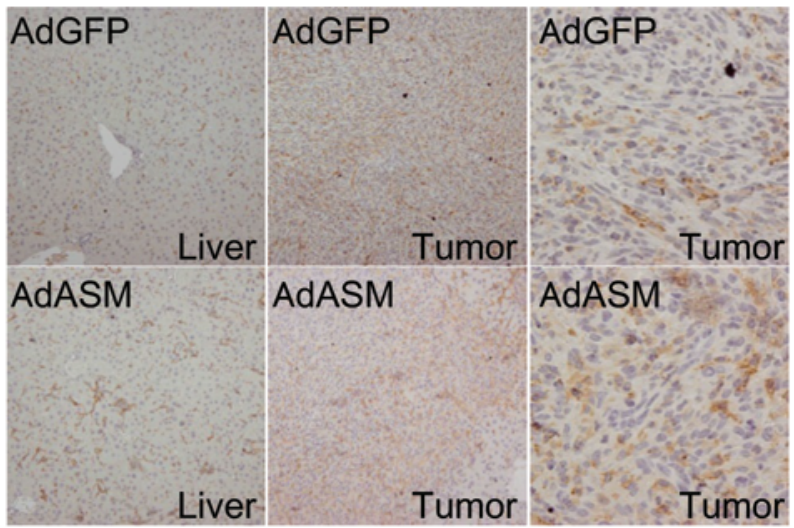

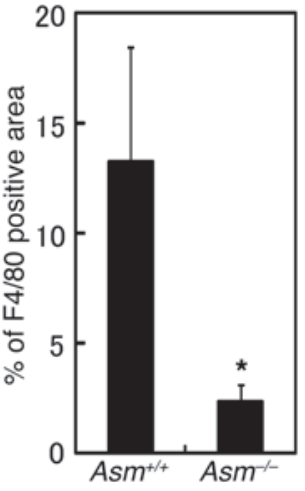

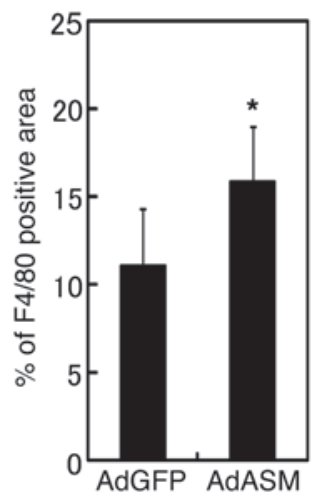

\section{Figure 2}

Macrophage depletion increased metastatic tumor growth in the liver. (A) GFP+ mice were intrasplenically injected with $2 \times 10^{4}$ SL4 cells. GFP fluorescence was visualized with fluorescent microscopy, and macrophages were detected in metastatic tumor and liver by immunofluorescent staining for $F 4 / 80$ (blue). Original magnification, $\times 400$. (B and C) $\mathrm{Asm}^{+/+}$and $\mathrm{Asm}^{-/-}$mice (B) and AdGFPand AdASM-infected wild-type mice (C) were intrasplenically injected with $2 \times 10^{4}$ SL4 cells. Expression of $F 4 / 80$ in the liver and metastatic tumor was examined by immunohistochemistry with an anti-F4/80 antibody to assess the number of macrophages. Original magnification, $\times 100$ (left and middle); $\times 400$ (right). (D) Wildtype mice were injected with SL4 cells and treated with PBS or alendronate before sacrifice 14 days after inoculation. Images of livers after excision and liver sections stained with H\&E (loupe magnification) are shown. Intrahepatic tumor load is presented as hepatic replacement area, based on measurement of 3 nonsequential sections. Results are mean $\pm S D$ of data collected from at least 5 independent experiments. ${ }^{*} P<0.05$, 2-tailed Student's $t$ test.

D
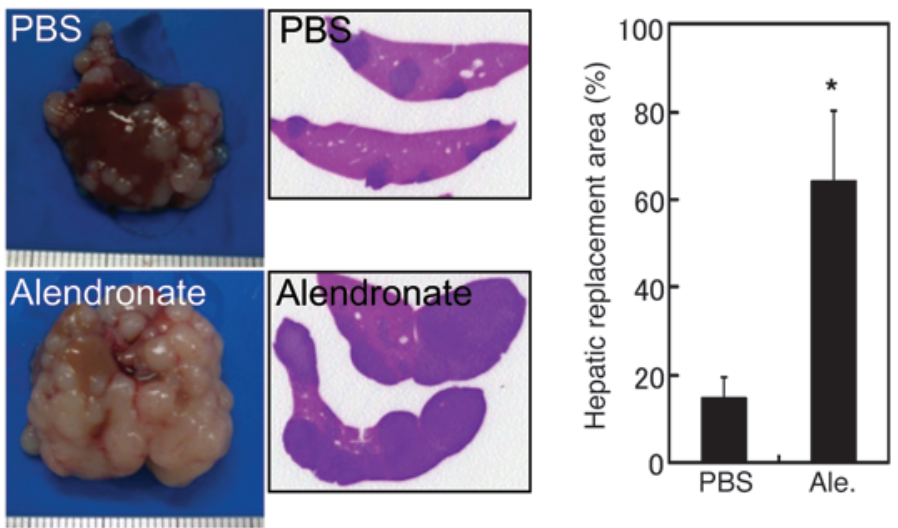
A
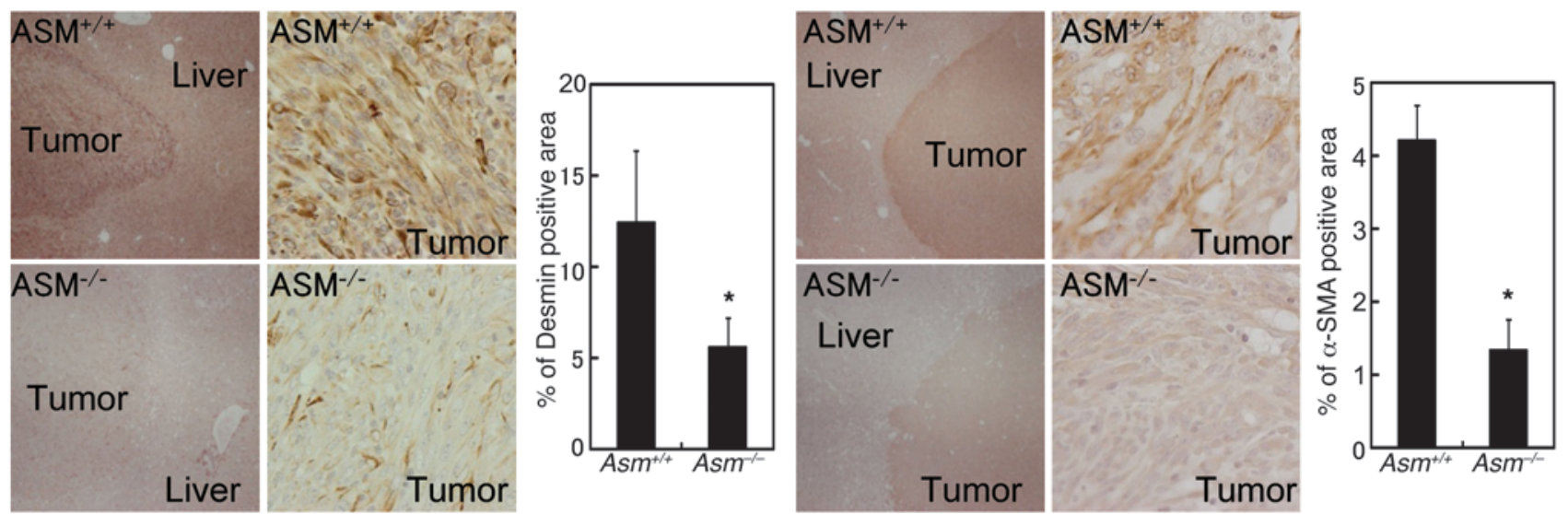

B
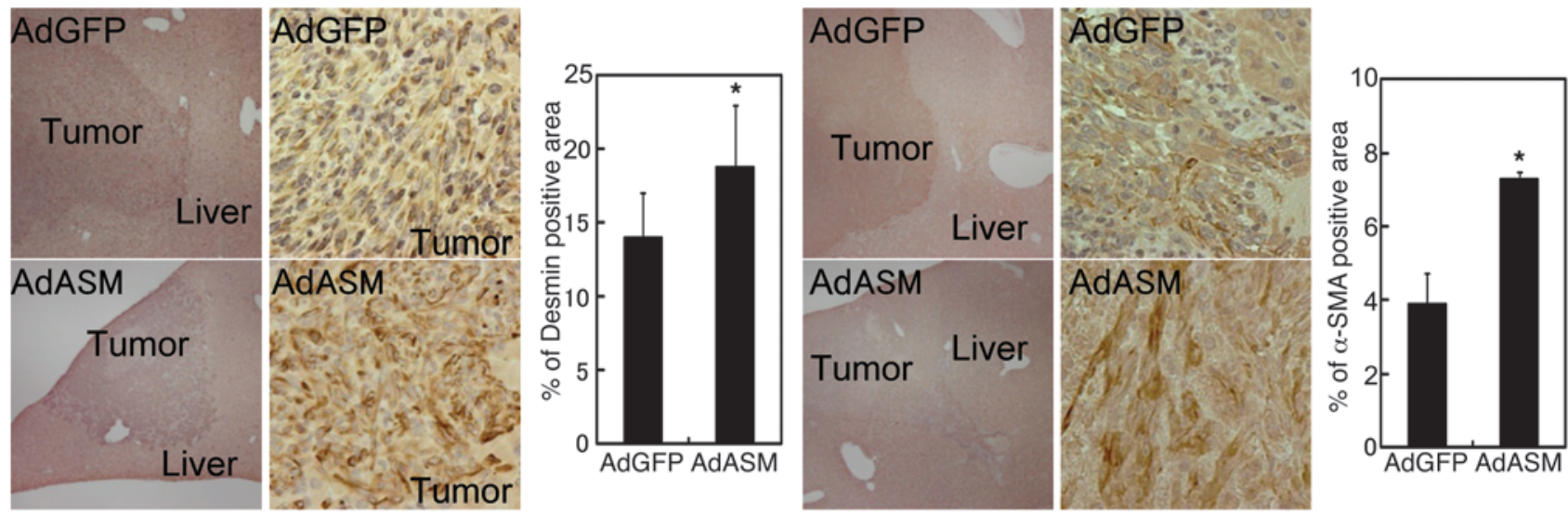

C
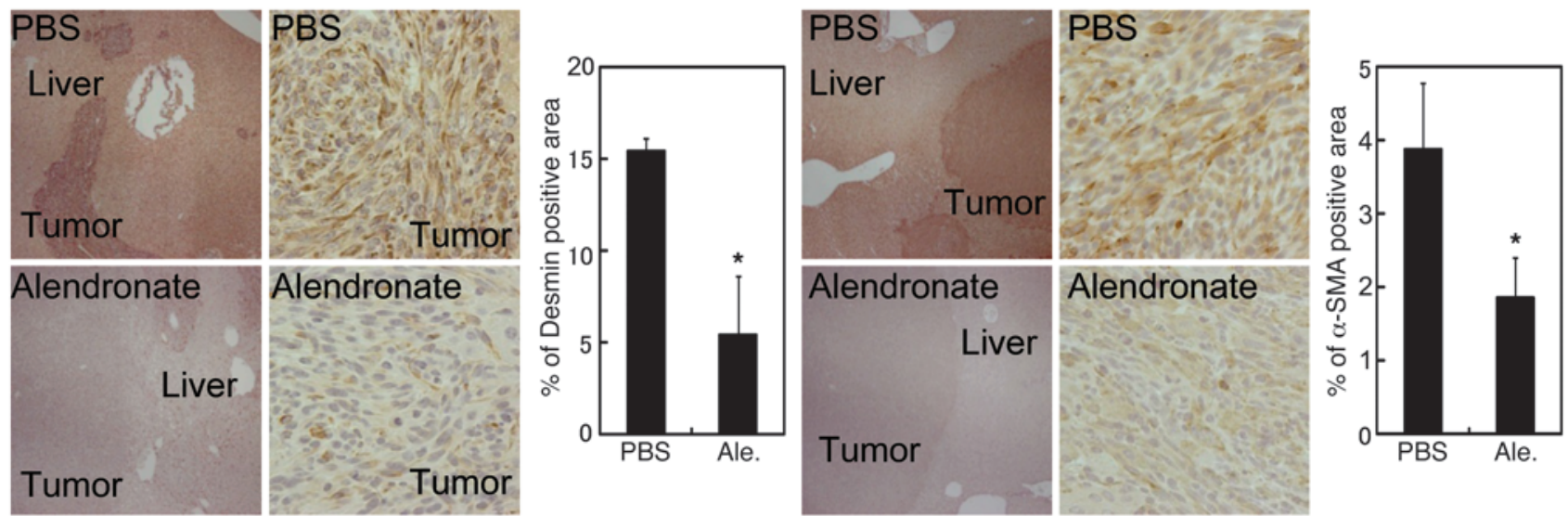

Figure 3

ASM deficiency or macrophage depletion decreased hMFs, and ASM overexpression increased hMFs, around the tumor invasive margin. Asm ${ }^{+/+}$ and $\mathrm{Asm}^{-/-}$mice (A), AdGFP- or AdASM-infected wild-type mice (B), and wild-type mice treated with PBS or alendronate (C) were intrasplenically injected with $2 \times 10^{4}$ SL4 cells and sacrificed 14 days after inoculation. Expression of desmin (left) and $\alpha$-SMA (right) around the tumor invasive margin was examined by immunohistochemistry, and measurement of immunostain-positive area was performed. Original magnification, $\times 40$ (left); $\times 400$ (right). Results are mean \pm SD of data collected from at least 5 independent experiments. ${ }^{*}<<0.05,2$-tailed Student's $t$ test.

has been reported that $\mathrm{S} 1 \mathrm{P}$ induces a switch from the M1 to the M2 macrophage subtype (18), in the present study, S1P did not alter the mRNA expression levels of M1 and M2 markers, which suggests that S1P does not affect the macrophage phenotype. Instead, our observations that $1 \mu \mathrm{M}$ S1P treatment for 72 hours increased Timp1
mRNA expression in isolated hMFs (Figure 5C) and that adenoviral overexpression of SphK1 (AdSphK) in hepatocytes prior to cancer cell inoculation inhibited tumor growth (Supplemental Figure 5A) suggest that ASM suppresses tumor growth via S1P production. The fold increase of Timp $1 \mathrm{mRNA}$ induced by S1P did not seem to 
A

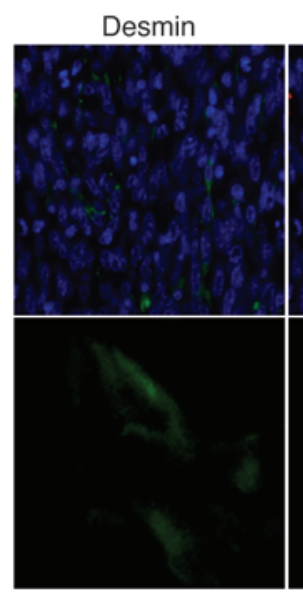

C

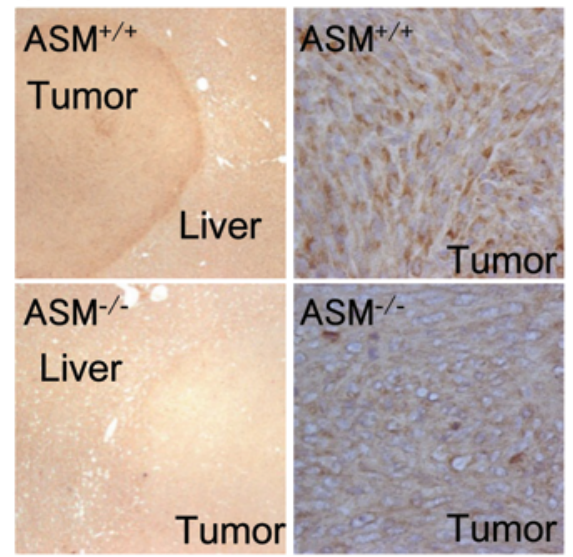

E

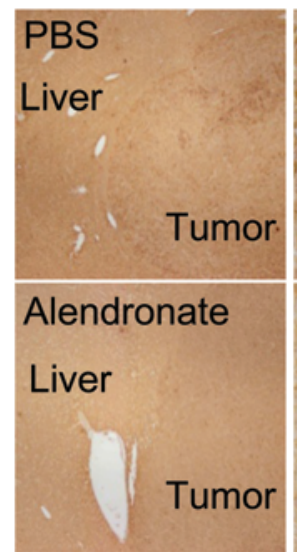

TIMP1

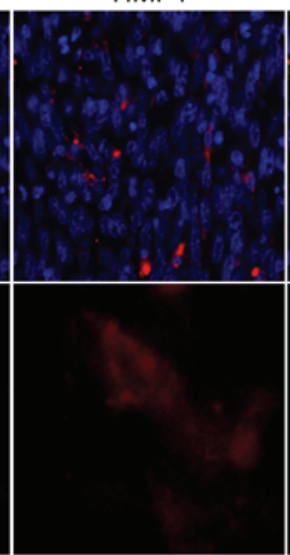

Merge

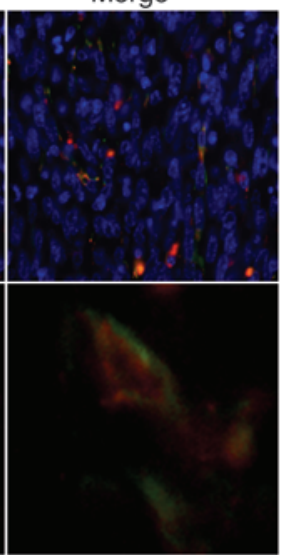

D

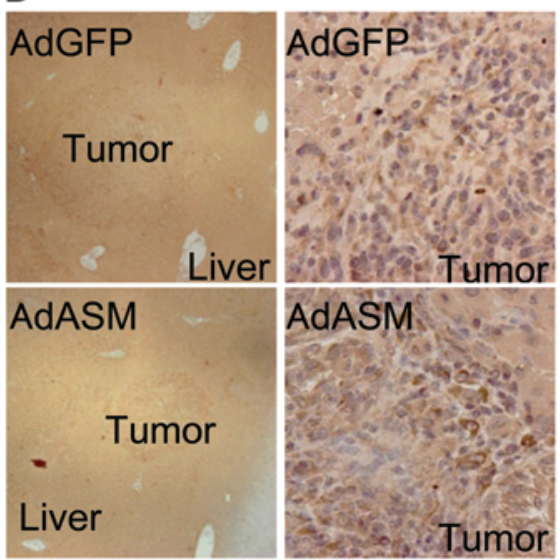

B

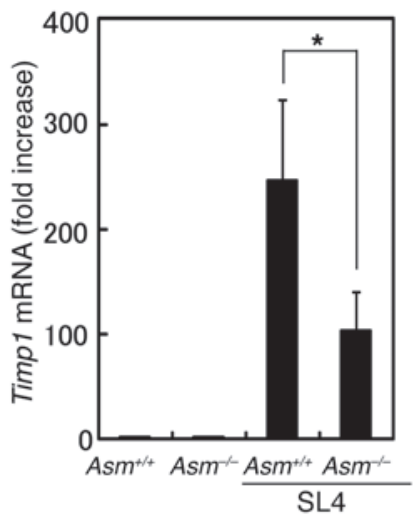

$\mathbf{F}$

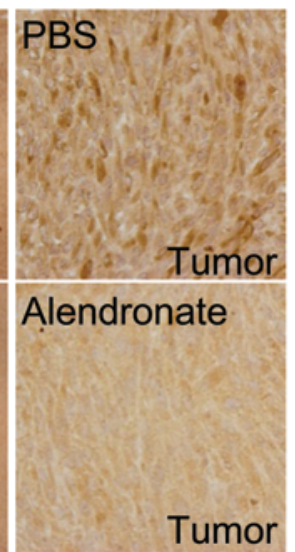

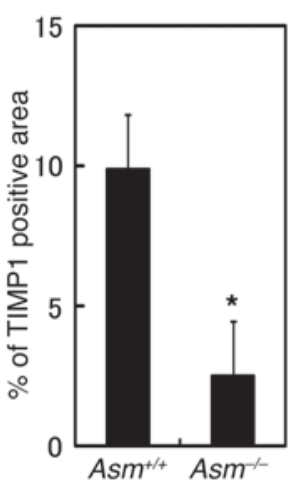

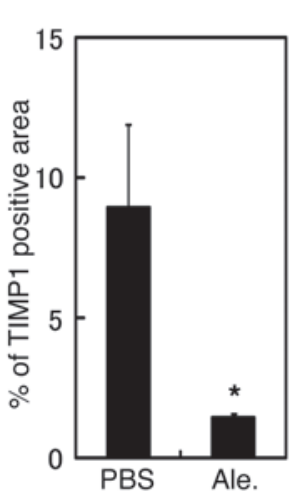

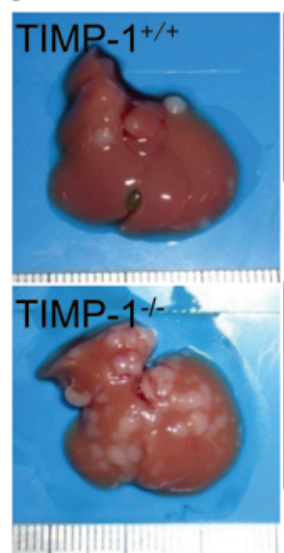
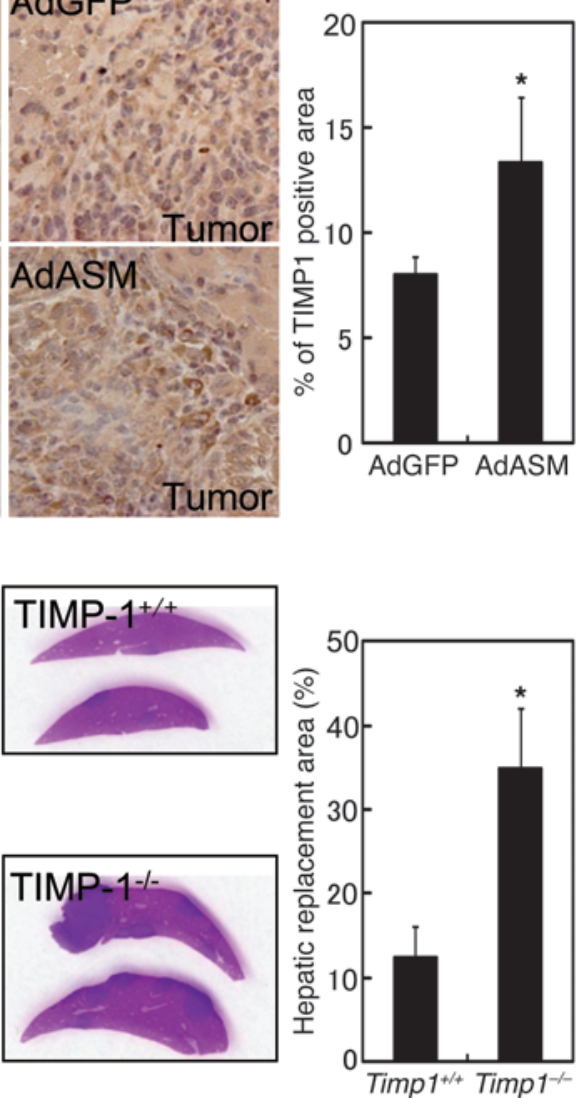

\section{Figure 4}

TIMP1 deficiency increased metastatic tumor growth in the liver. (A) Wild-type mice were intrasplenically injected with $2 \times 10^{4}$ SL4 cells and sacrificed 14 days after inoculation. Expression of desmin (green) and TIMP1 (red) around the invasive margin of the tumors was examined by immunofluorescent staining. Nuclei were stained with DAPI (blue, top). Original magnification, $\times 400$ (top); $\times 1,600$ (bottom). (B) Asm ${ }^{+/+}$and Asm $^{-/}$mice were intrasplenically injected with $5 \times 10^{5} \mathrm{SL} 4$ cells and sacrificed 7 days after inoculation. Timp1 mRNA expression in the liver was determined by quantitative real-time RT-PCR. (C-E) $\mathrm{Asm}^{+/+}$and $\mathrm{Asm}^{-/-}$mice (C), AdGFP- or AdASM-infected wild-type mice (D), and wild-type mice treated with PBS or alendronate (E) were intrasplenically injected with $2 \times 10^{4}$ SL4 cells and sacrificed 14 days after inoculation. TIMP1 expression around the tumor invasive margin was examined by immunohistochemistry, and measurement of TIMP1+area was performed. Original magnification, $\times 40$ (left); $\times 400$ (right). (F) Timp1+/+ and Timp1-/- mice were intrasplenically injected with $2 \times 10^{4}$ SL4 cells and sacrificed 14 days after inoculation. Images of livers after excision and liver sections stained with H\&E (loupe magnification) are shown. Intrahepatic tumor load is presented as hepatic replacement area, based on measurement of 3 nonsequential sections. Results are mean \pm SD of data collected from at least 5 independent experiments. ${ }^{*} P<0.05$, 2-tailed Student's $t$ test. 

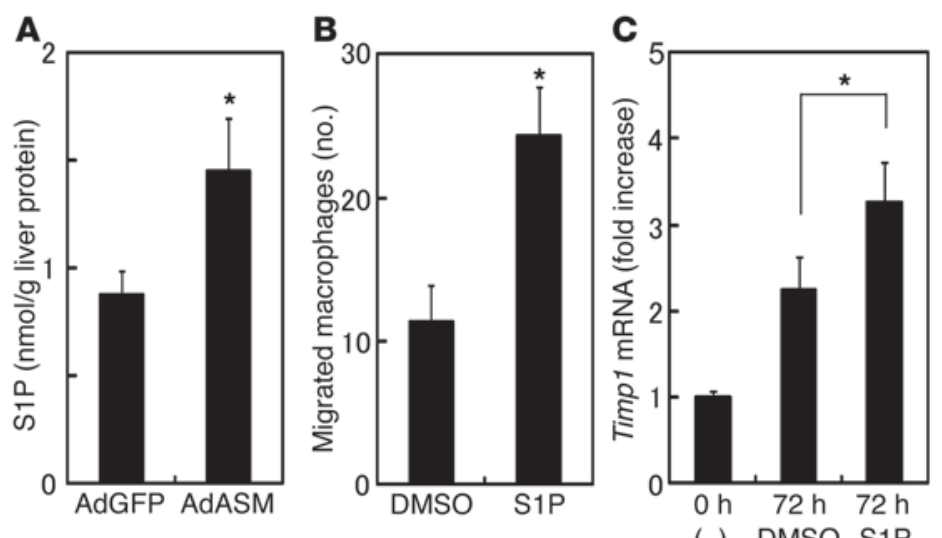

\section{D}

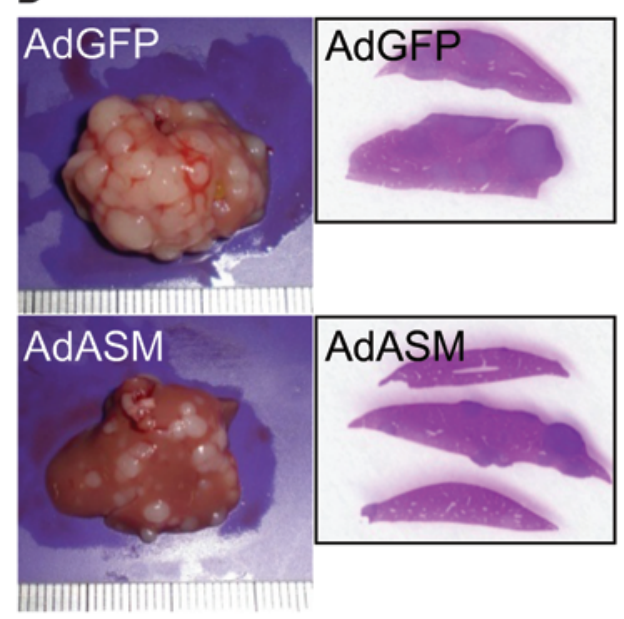

\section{Figure 5}

S1P promoted macrophage migration and Timp1 mRNA expression in hMFs. (A) Wild-type mice were infected with AdGFP or AdASM, and S1P was examined by MS analysis 3 days after infection. (B) After addition of CD11 beritoneal macrophages and $1 \mu \mathrm{M}$ S1P to the upper and lower chambers, respectively, of transwell plates, cells were incubated for 4 hours. The number of macrophages that migrated to the underside of the chamber was determined. (C) Primary rat hMFs were incubated with or without $1 \mu \mathrm{M}$ S1P on plastic dishes for 72 hours before determination of Timp1 mRNA levels by quantitative real-time RT-PCR. (D) Wild-type mice were intrasplenically injected with $2 \times 10^{4}$ SL4 cells, infected with AdGFP or AdASM 5 days after inoculation, and sacrificed 14 days after infection (i.e., 19 days after inoculation). Images of livers after excision and liver sections stained with H\&E (loupe magnification) are shown. Intrahepatic tumor load is presented as hepatic replacement area, based on measurement of 3 nonsequential sections. Results are mean \pm SD of data collected from 4 (A) or at least 5 (B-D) independent experiments. ${ }^{*} P<0.05,2$-tailed Student's $t$ test. be large (Figure 5C). However, a 48-hour treatment with TGF- $\beta$ has previously been reported to induce an approximately 2 -fold increase of TIMP1 mRNA in cultured human hMFs (34). This suggests that the induction of Timp1 mRNA by S1P was not a small change, and it is possible that the induction might be sufficient. $5 \mu \mathrm{M} \mathrm{S1P}$ induced TIMP1 expression to a similar degree as that observed using $1 \mu \mathrm{M}$ $\mathrm{S} 1 \mathrm{P}$ (data not shown), which suggests that $1 \mu \mathrm{M}$ S1P fully induces activation of $\mathrm{S} 1 \mathrm{P}$ receptors.

As described above, ASM exerted an antitumor effect through macrophage accumulation and TIMP1 production from hMFs via S1P. To evaluate AdASM administration as a form of treatment against metastatic liver tumors of colon cancer, AdASM was administered to mice 5 days after SL4 cell inoculation. AdASM suppressed tumor growth, even when it was administered after cell inoculation (Figure 5D). However, AdSphK administration did not suppress tumor growth (Supplemental Figure 5B), although the accumulation of $\mathrm{F} 4 / 80^{+}$and desmin ${ }^{+}$cells was similar to that in AdASM-treated mice (Supplemental Figure 6A). In addition, accumulation of $\mathrm{F} 4 / 80^{+}$and desmin ${ }^{+}$cells was observed to be equal, regardless of whether mice were infected with AdSphK before or after SL4 cell inoculation (data not shown). Adenovirus-mediated GFP expression was observed in the tumors when the virus was administered after cell inoculation (Supplemental Figure 6B), which suggests that the adenovirus infects not only hepatocytes, but also inoculated SL4 cells. The observation that AdSphK increased proliferation of cultured SL4 cells (Supplemental
Figure 6C) indicated that the antitumor effects of overexpressed SphK1 in the liver may be counteracted by the tumor-promoting effects of SphK1 on SL4 cell proliferation.

\section{Discussion}

In the present study, we investigated the contribution of ASM to the suppression of tumor growth in liver metastasis of colon cancer cells. Our results indicated that liver ASM inhibits tumor growth through S1P formation and subsequent macrophage accumulation and TIMP1 production from hMFs. These results suggest novel therapeutic possibilities for treating metastatic liver tumors of colon cancer.

ASM in cancer cells is involved in cell death and plays an important role in the host response to a variety of anticancer treatments, including those for colon cancer (1). In the present study, metastatic tumors of colon cancer cells stimulated ASM expression and ceramide in liver cells. Moreover, host ASM deficiency increased, while overexpression of ASM in the liver decreased, the growth of liver tumors after inoculation with colon cancer cells, which suggests that ASM in host cells contributes to antitumor defense. Among the roles that ASM plays in liver cells, ASM in hepatocytes stimulates glucose uptake, resulting in improvement of glucose tolerance in mice through S1P production (27). ASM has pleiotropic signaling functions related to posttranslational processing of ASM, and secretory and lysosomal ASM (35) may have distinct functions. Although it is unclear which type of ASM was 
involved in S1P production in our study, hepatocytes represented one source of S1P, as the S1P elevation by AdASM was inhibited in both SphK1-deficient livers and SphK1-deficient primary cultured rat hepatocytes (data not shown). Previous studies have shown that the generated S1P can be secreted and act as a ligand for S1P receptors (S1PRs; refs. 27, 36, 37), while the S1P/S1PR1 axis controls the trafficking and migration of immune cells, including macrophages $(16,17,36)$. In accordance with these prior reports, $\mathrm{S} 1 \mathrm{P}$ increased the migration of peritoneal macrophages in vitro, with less and more macrophage accumulation, respectively, in the tumors of $\mathrm{Asm}^{-1-}$ and AdASM-infected mice compared with controls. These findings indicate that ASM in hepatocytes leads to macrophage accumulation in tumors via S1P production and that this process exerts an antitumor effect, in contrast to macrophage depletion. Although it has been reported that TAMs, which resemble regulatory M2 macrophages $(13,14)$, function as promoters of tumor progression (7-9), the macrophages in the tumors inoculated with SL4 colon cancer cells in this study were M1 dominant. It has been proposed that a gradual shift of TAM polarization from the M1 to the M2 subtype occurs during different stages of tumor progression, due to dynamic changes in the tumor microenvironment (38), and is paralleled by the gradual inhibition of NF- $\mathrm{KB}$ (39). In this study, we observed immunofluorescent staining of $\mathrm{NF}-\kappa \mathrm{B}$ p 65 in the nuclei of $\mathrm{F} 4 / 80^{+}$cells in the tumors (data not shown), which suggests that NF-кB is activated in these macrophages. This finding may indicate that in metastatic liver tumors characterized by rapid growth, such as by increased numbers of SL4 cells (40), the accumulated TAMs may retain an M1 phenotype that displays a cytotoxic proinflammatory phenotype. Thus, the number of macrophages present in the tumor is inversely correlated with tumor growth.

Myofibroblasts represent another important component of the tumor stroma; these cells are driven by migration and proliferation-stimulating factors released from cancer cells. Whereas some studies have reported a supportive role for myofibroblasts in cancer cell development (41) and a direct correlation between their presence and poor prognosis (42), others have reported a protective role of myofibroblasts against cancer cells $(43,44)$, with p53 in stromal fibroblasts demonstrated to inhibit tumor growth and malignant progression (45). In the current study using SL4 cell inoculation, hMF accumulation was found to be dependent on macrophage accumulation and inversely correlated with tumor growth. Specifically, hMF accumulation was increased only around the invasive margins of the tumors, and no hMF infiltration or collagen deposition was observed in the central part of tumors (data not shown). These findings indicate that the supportive effects of hMFs in tumor growth may be limited. However, the observation of enhanced tumor growth in Timp $1^{-1-}$ mice indicates that TIMP1 produced from hMFs, stimulated by S1P, might inhibit tumor growth through inhibition of ECM degradation. Although previous studies reported a protumor role for TIMP1, TIMP1 staining was observed only around the tumor invasive margin in the current study, which indicates that the supportive effects of TIMP1 in tumor growth may also be limited.

Whereas ceramide acts as a signaling molecule in the induction of cell responses, such as apoptosis and growth arrest $(3,46,47)$, $\mathrm{S} 1 \mathrm{P}$ acts as a potent mitogen for a variety of cell types (48), with SphK1/S1P contributing to colon carcinogenesis (49). Ceramide levels in colon carcinoma tissue are significantly lower than those in normal tissue (50), reflecting the fact that the dynamic balance between intracellular levels of ceramide and S1P (the "ceramide/ S1P rheostat") determines cell growth and cell death in colon cancer. In the present study, adenoviral overexpression of SphK1 in the liver inhibited tumor growth when colon cancer cells were inoculated after infection. In contrast to AdASM, AdSphK did not exert an inhibitory effect when administered after cell inoculation, which suggests that its inhibitory effect on existing tumors is less than that of AdASM. The observation that AdSphK increased the proliferation of cultured SL4 cells suggests that it decreased the ceramide/S1P ratio in the cancer cells, which may promote their proliferation. In contrast, AdASM might increase the ceramide/ $\mathrm{S} 1 \mathrm{P}$ ratio, which may inhibit SL4 cell proliferation. We therefore conclude that AdASM, but not AdSphK, may be a useful candidate for gene therapy against metastatic liver tumors of colon cancer.

In $\mathrm{Asm}^{-/-}$mice, B16C2M melanoma cells formed small metastatic lesions in the liver (4 of 9, 44.4\%) whereas none of the $\mathrm{Asm}^{+/+}$ mice developed metastases ( 0 of $11,0 \%$; Supplemental Figure 7 ), which suggests that the antitumor effect of ASM is not specific for colon cancer cells. However, use of the Asm knockout may have secondary effects due to the severe lipid storage defect in these mice, particularly in macrophages. Asm ${ }^{-/-}$mice are known to have severe immune defects $(51,52)$ and display severe neurocutaneous disease by 8 weeks of age. Thus, $A s \mathrm{~m}^{-/-}$mice may have various abnormalities in the liver as a normal pathology. In our study, the livers of $\mathrm{Asm}^{-/-}$mice without injection of SL4 cells did not show the nodular appearance. In addition, liver weight of $A \mathrm{sm}^{-/-}$mice without injection of SL4 cells was comparable to that of $\mathrm{Asm}^{+/+}$ mice. Thus, it seems unlikely that the changes are due to normal Asm ${ }^{-1-}$ mouse pathology. In addition, besides ASM, ceramide synthesized by the de novo pathway can be involved in TIMP1 regulation. These data do not provide direct evidence that S1P production in hepatocytes recruits macrophages, and experiments using mice with conditional knockout of SphK are also required to confirm the involvement of SphK and S1P in macrophage recruitment and TIMP1 regulation. Moreover, the mechanism by which tumor cells stimulate ASM expression in hepatocytes remains unclear, and there is no direct evidence of a positive correlation between ASM activity in hepatocytes and TIMP1 expression in hMFs; further studies are needed to resolve these uncertainties. In conclusion, we found here that ASM in hepatocytes inhibited tumor growth via S1P formation and subsequent cytotoxic macrophage accumulation. This S1P formation in turn stimulated TIMP1 production by hMFs, leading to tumor suppression. Thus, targeting ASM may represent a new therapeutic strategy for treating liver metastasis of colon cancer.

\section{Methods}

Animals. Asm ${ }^{-1-}$ mice (C57BL/6 background; ref. 47) were bred for use in this study. GFP mice (C57BL/6 background), which express EGFP ubiquitously under the CAG promoter (chicken $\beta$-actin promoter, rabbit $\beta$-globin poly A, CMV-IE enhancer), were obtained from Riken Bio Resource Center. Timp $1^{-1-}$ mice (C57BL/6 background) were obtained from Jackson Laboratory. Male Wistar rats and female C57BL/6J wild-type mice were obtained from Japan SLC. GFP ${ }^{+} A s m^{-/-}$mice were generated for this study.

Cell culture. A red fluorescent protein-expressing mouse colon adenocarcinoma cell line (SL4; Anti-Cancer Japan, Osaka, Japan) was maintained as a monolayer culture in RPMI-1640 (Invitrogen) containing 10\% FBS supplemented with penicillin and streptomycin (Invitrogen). Cells were harvested using trypsin and EDTA, washed with PBS, and then resuspended in serum-free RPMI-1640 $\left(2 \times 10^{5}\right.$ or $5 \times 10^{6}$ cells $\left./ \mathrm{ml}\right)$. 
Liver metastasis model. Female wild-type (C57BL/6J) mice aged 8-10 weeks were divided into 2 groups. After a small incision was made under anesthesia to expose the spleen, $0.1 \mathrm{ml}$ of a viable cell suspension containing $5 \times 10^{5}$ cells/mouse was injected into the spleen of group 1 , and the same suspension containing $2 \times 10^{4}$ cells/mouse was injected into group 2 . Mice were sacrificed on day 7 (group 1) and day 14 (group 2) after cell inoculation. The livers of group 1 were immediately removed, washed in ice-cold PBS, and weighed, after which a part of the dissected liver tissue was frozen in liquid nitrogen. For assessment of liver metastatic tumors in group 2, the intrahepatic tumor was scored as the hepatic replacement area and maximum diameter of tumor, based on examination of 3 nonsequential $\mathrm{H} \&$ E-stained sections using ImageJ software (NIH). For detection of GFP fluorescence, livers were first perfused in situ with $0.5 \mathrm{mM}$ EGTA containing salt solution to remove peripheral blood cells. The livers were then perfused with $4 \%$ paraformaldehyde, fixed with $4 \%$ paraformaldehyde for 24 hours, soaked in $15 \%$ sucrose in PBS for 12 hours, soaked in $30 \%$ sucrose for 24 hours at $4{ }^{\circ} \mathrm{C}$ under constant agitation, and then embedded in OCT compound to cut sections on a cryostat. The $\mathrm{GFP}^{+}$area was determined using Image J software and shown as a percentage of the total section area.

Histological analysis. F4/80, desmin, $\alpha$-SMA, and TIMP1 were stained with anti-F4/80 (Santa Cruz Biotechnology), anti-desmin (Lab Vision), anti$\alpha$-SMA (clone 1A4; Sigma-Aldrich), and anti-TIMP1 (R\&D Systems) antibodies, respectively, using Vectastain Elite ABC Kit or M.O.M Immunodetection Kit (Vector Laboratories). Diaminobenzidine tetrahydrochloride was used as peroxidase substrate, and sections were counterstained with hematoxylin. The immunostain-positive area was determined using Image J software and shown as a percentage of the total section area. In some experiments, fluorescent dye-labeled secondary antibodies (Alexa Fluor 350 antirat for F4/80, Alexa Fluor 488 anti-rabbit for desmin, and Alexa Fluor 594 anti-goat for TIMP1) were used for detection of primary antibodies.

Depletion of macrophages. Liposome-encapsulated alendronate (Sigma-Aldrich) was generated as previously reported (53). The alendronate was injected in the mice 4 times: 1 day before cell inoculation and 4, 7, and 10 days after. Liposome-encapsulated PBS was injected into the mice as a control.

Recombinant adenoviruses. Mice were infected with 2 adenoviruses $\left(5 \times 10^{8} \mathrm{pfu} /\right.$ mouse) by intravenous injection 1 day before or 5 days after cell inoculation. Of the 2 adenovirus- 5 variants, AdGFP expresses GFP, whereas AdASM expresses both ASM and GFP, because it contains a GFP sequence (27).

Mass spectrometric (MS) analysis of S1P. Electrospray ionization MS/MS analysis was performed using a Thermo-Finnigan TSQ 7000 Triple Quadrupole Mass Spectrometer (GenTEch Scientific Inc.) in multiple-reaction monitoring positive ionization mode, as previously reported (54). S1P level was normalized by total protein per sample.

Macrophage migration assay. For isolation of peritoneal macrophages, wildtype mice were injected intraperitoneally with cold normal saline. Peritoneal macrophages were purified using MACS Cell Separation Columns with CD11b microbeads (Miltenyi Biotec) from the lavage fluid. The cell migra- tion assay was performed using 24 -well transwell plates $(5-\mu \mathrm{m}$ pore size; Costar). The peritoneal macrophages $\left(1 \times 10^{5}\right.$ cells $)$ in DMEM containing $1 \%$ BSA were loaded into the upper chambers, while the lower chambers were filled with medium with or without $1 \mu \mathrm{M} \mathrm{S1P}$ (Matreya LLC). Cells were incubated for 4 hours, then the migrated macrophages on the underside of the chambers were fixed and stained with Giemsa solution (Wako). Migration was expressed as the number of cells per field.

Quantitative real-time RT-PCR. Rat primary liver hMFs were isolated as previously described (25). The cells were cultured in DMEM with $10 \%$ FBS on plastic dishes for 4 hours and administered S1P or vehicle $(1 \mu \mathrm{M})$, and RNA was extracted 0 or 72 hours after S1P stimulation. The RNeasy and DNase Kits (Qiagen) was used for RNA extraction from liver tissue and cultured cells, and the High-Capacity cDNA Reverse Transcription Kit (Applied Biosystems) was used for reverse transcription. Quantitative real-time RT-PCR was performed using the SYBR Premix Ex Taq (Takara) for Timp1 (forward, TGGGGAACCCATGAATTTAG; reverse, TCTGGCATCCTCTTGTTGC) and probe and primer sets (Applied Biosystems) for $18 \mathrm{~S}$ with LightCycler 480 (Roche Applied Science). The changes were normalized based on $18 \mathrm{~S}$ rRNA values.

Statistics. Data are expressed as mean \pm SD of data collected from 5 independent experiments. Data between groups were analyzed by 2-tailed Student's $t$ test. A $P$ value less than 0.05 was considered significant.

Study approval. All experiments were conducted in accordance with the institutional guidelines of Gifu University and were approved by the animal research committee and the committee on living modified organisms of Gifu University.

\section{Acknowledgments}

This work was supported by grants from the Takeda Science Foundation, the Kondou Kinen Medical Foundation, the Kurozumi Medical Foundation, the Yasuda Medical Foundation, and the Senshin Medical Research Foundation and by Grants-inAid for Scientific Research from the Ministry of Education, Science, Sports, and Culture of Japan (23790787, 24890081, and 22590726). We thank Jacek Bielawski for conducting the sphingolipid measurement and Yoshiko Banno for performing the measurement of ASM activity. B16C2M cells were provided by the Cell Resource Center for Biomedical Research, Institute of Development, Aging and Cancer, Tohoku University (Sendai, Japan).

Received for publication June 12, 2012, and accepted in revised form November 13, 2012.

Address correspondence to: Yosuke Osawa, Department of Pharmacology, Gifu University Graduate School of Medicine, 1-1 Yanagido Gifu 501-1194, Japan. Phone: 81.58.230.6217; Fax: 81.58.230.6218; E-mail: osawa-gif@umin.ac.jp.
1. Smith EL, Schuchman EH. The unexpected role of acid sphingomyelinase in cell death and the pathophysiology of common diseases. FASEB J. 2008; 22(10):3419-3431.

2. Jenkins RW, Canals D, Hannun YA. Roles and regulation of secretory and lysosomal acid sphingomyelinase. Cell Signal. 2009;21(6):836-846.

3. Hannun YA. Functions of ceramide in coordinating cellular responses to stress. Science. 1996; 274(5294):1855-1859.

4. Furuya H, Shimizu Y, Kawamori T. Sphingolipids in cancer. Cancer Metastasis Rev. 2011;30(3-4):567-576.

5. Nemoto $S$, et al. Sphingosine kinase isoforms regulate oxaliplatin sensitivity of human colon cancer cells through ceramide accumulation and Akt activation. J Biol Chem. 2009;284(16):10422-10432.
6. Coussens LM, Werb Z. Inflammation and cancer. Nature. 2002;420(6917):860-867.

7. Hagemann T, Biswas SK, Lawrence T, Sica A, Lewis CE. Regulation of macrophage function in tumors: the multifaceted role of NF-кB. Blood. 2009; 113(14):3139-3146.

8. Condeelis J, Pollard JW. Macrophages: obligate partners for tumor cell migration, invasion, and metastasis. Cell. 2006;124(2):263-266.

9. Mantovani A, Allavena P, Sica A, Balkwill F. Cancer-related inflammation. Nature. 2008; 454(7203):436-444.

10. Bingle L, Brown NJ, Lewis CE. The role of tumourassociated macrophages in tumour progression: implications for new anticancer therapies.J Pathol. 2002;196(3):254-265.
11. Zhou Q, et al. The density of macrophages in the invasive front is inversely correlated to liver metastasis in colon cancer. J Transl Med. 2010;8:13.

12. Forssell J, et al. High macrophage infiltration along the tumor front correlates with improved survival in colon cancer. Clin Cancer Res. 2007; 13(5):1472-1479.

13. Mosser DM, Edwards JP. Exploring the full spectrum of macrophage activation. Nat Rev Immunol. 2008; 8(12):958-969.

14. Balkwill F, Charles KA, Mantovani A. Smoldering and polarized inflammation in the initiation and promotion of malignant disease. Cancer Cell. 2005; 7(3):211-217.

15. Ma J, et al. The M1 form of tumor-associated macrophages in non-small cell lung cancer is posi- 
tively associated with survival time. BMC Cancer. 2010;10:112.

16. Keul P, et al. Sphingosine-1-phosphate receptor 3 promotes recruitment of monocyte/macrophages in inflammation and atherosclerosis. Circ Res. 2011; 108(3):314-323.

17. Rivera J, Proia RL, Olivera A. The alliance of sphingosine-1-phosphate and its receptors in immunity. Nat Rev Immunol. 2008;8(10):753-763.

18. Weigert A, et al. Tumor cell apoptosis polarizes macrophages role of sphingosine-1-phosphate. $\mathrm{Mol}$ Biol Cell. 2007;18(10):3810-3819.

19. Keul P, et al. The sphingosine-1-phosphate analogue FTY720 reduces atherosclerosis in apolipoprotein E-deficient mice. Arterioscler Thromb Vasc Biol. 2007;27(3):607-613.

20. Wagenaar-Miller RA, Gorden L, Matrisian LM. Matrix metalloproteinases in colorectal cancer: is it worth talking about? Cancer Metastasis Rev. 2004; 23(1-2):119-135.

21. Elezkurtaj S, et al. Adenovirus-mediated overexpression of tissue inhibitor of metalloproteinases-1 in the liver: efficient protection against T-cell lymphoma and colon carcinoma metastasis. J Gene Med. 2004;6(11):1228-1237.

22. Martin DC, Ruther U, Sanchez-Sweatman $\mathrm{OH}$, Orr FW, Khokha R. Inhibition of SV40 T antigeninduced hepatocellular carcinoma in TIMP-1 transgenic mice. Oncogene. 1996;13(3):569-576.

23. Kruger A, Fata JE, Khokha R. Altered tumor growth and metastasis of a T-cell lymphoma in Timp-1 transgenic mice. Blood. 1997;90(5):1993-2000.

24. Jiang Y, Goldberg ID, Shi YE. Complex roles of tissue inhibitors of metalloproteinases in cancer. Oncogene. 2002;21(14):2245-2252.

25. Osawa Y, Hannun YA, Proia RL, Brenner DA. Roles of AKT and sphingosine kinase in the antiapoptotic effects of bile duct ligation in mouse liver. Hepatology. 2005;42(6):1320-1328.

26. Li C, et al. Sphingosine 1-phosphate (S1P)/S1P receptors are involved in human liver fibrosis by action on hepatic myofibroblasts motility. J Hepatol. 2011;54(6):1205-1213.

27. Osawa Y, et al. Acid sphingomyelinase regulates glucose and lipid metabolism in hepatocytes through AKT activation and AMP-activated protein kinase suppression. FASEB J. 2011;25(4):1133-1144.

28. Kan Z, et al. In vivo microscopy of hepatic metastases: dynamic observation of tumor cell invasion and interaction with Kupffer cells. Hepatology. 1995;21(2):487-494.

29. Klimp AH, de Vries EG, Scherphof GL, Daemen T. A potential role of macrophage activation in the treatment of cancer. Crit Rev Oncol Hematol. 2002; 44(2):143-161.

30. Caignard A, et al. Role of macrophage in the defense against intestinal cancers. Comp Immunol Microbiol Infect Dis. 1985;8(2):147-157.

31. Bayon LG, et al. Role of Kupffer cells in arresting circulating tumor cells and controlling metastatic growth in the liver. Hepatology. 1996;23(5):1224-1231.

32. Holten-Andersen MN, et al. Localization of tissue inhibitor of metalloproteinases 1 (TIMP-1) in human colorectal adenoma and adenocarcinoma. Int J Cancer. 2005;113(2):198-206.

33. Granado MH, et al. Ceramide 1-phosphate (C1P) promotes cell migration Involvement of a specific C1P receptor. Cell Signal. 2009;21(3):405-412.

34. Schulze-Krebs A, et al. Hepatitis C virus-replicating hepatocytes induce fibrogenic activation of hepatic stellate cells. Gastroenterology. 2005;129(1):246-258.

35. Schissel SL, Keesler GA, Schuchman EH, Williams KJ, Tabas I. The cellular trafficking and zinc dependence of secretory and lysosomal sphingomyelinase, two products of the acid sphingomyelinase gene. J Biol Chem. 1998;273(29):18250-18259.

36. Spiegel S, Milstien S. The outs and the ins of sphingosine-1-phosphate in immunity. Nat Rev Immunol. 2011;11(6):403-415.

37. Osawa Y, et al. TNF- $\alpha$-induced sphingosine 1-phosphate inhibits apoptosis through a phosphatidylinositol 3-kinase/Akt pathway in human hepatocytes. J Immunol. 2001;167(1):173-180.

38. Sica A, Bronte V. Altered macrophage differentiation and immune dysfunction in tumor development. J Clin Invest. 2007;117(5):1155-1166.

39. Dunn GP, Bruce AT, Ikeda H, Old LJ, Schreiber RD. Cancer immunoediting: from immunosurveillance to tumor escape. Nat Immunol. 2002;3(11):991-998.

40. Morimoto-Tomita M, Ohashi Y, Matsubara A, Tsuiji M, Irimura T. Mouse colon carcinoma cells established for high incidence of experimental hepatic metastasis exhibit accelerated and anchorage-independent growth. Clin Exp Metastasis. 2005; 22(6):513-521.

41. De Wever $O$, et al. Tenascin-C and SF/HGF produced by myofibroblasts in vitro provide convergent pro-invasive signals to human colon can- cer cells through RhoA and Rac. FASEB J. 2004; 18(9):1016-1018

42. Tsujino T, et al. Stromal myofibroblasts predict disease recurrence for colorectal cancer. Clin Cancer Res. 2007;13(7):2082-2090.

43. Barsky SH, Gopalakrishna R. Increased invasion and spontaneous metastasis of BL6 melanoma with inhibition of the desmoplastic response in C57 BL/6 mice. Cancer Res. 1987;47(6):1663-1667.

44. Peyrol S, et al. Lysyl oxidase gene expression in the stromal reaction to in situ and invasive ductal breast carcinoma. Am J Pathol. 1997;150(2):497-507.

45. Moskovits N, Kalinkovich A, Bar J, Lapidot T, Oren M. p53 Attenuates cancer cell migration and invasion through repression of SDF-1/CXCL12 expression in stromal fibroblasts. Cancer Res. 2006; 66(22):10671-10676.

46. Pettus BJ, Chalfant CE, Hannun YA. Ceramide in apoptosis: an overview and current perspectives. Biochim Biophys Acta. 2002;1585(2-3):114-125.

47. Osawa Y, et al. Roles for C16-ceramide and sphingosine 1-phosphate in regulating hepatocyte apoptosis in response to tumor necrosis factor- $\alpha$. J Biol Chem. 2005;280(30):27879-27887.

48. Spiegel S, Milstien S. Sphingosine-1-phosphate: an enigmatic signalling lipid. Nat Rev Mol Cell Biol. 2003;4(5):397-407.

49. Kawamori $\mathrm{T}$, et al. Role for sphingosine kinase 1 in colon carcinogenesis. FASEB J. 2009;23(2):405-414.

50. Selzner $M$, et al. Induction of apoptotic cell death and prevention of tumor growth by ceramide analogues in metastatic human colon cancer. Cancer Res. 2001;61(3):1233-1240.

51. Kuemmel TA, Thiele J, Schroeder R, Stoffel W. Pathology of visceral organs and bone marrow in an acid sphingomyelinase deficient knock-out mouse line, mimicking human Niemann-Pick disease type A. A light and electron microscopic study. Pathol Res Pract. 1997;193(10):663-671.

52. Horinouchi K, et al. Acid sphingomyelinase deficient mice: a model of types A and B Niemann-Pick disease. Nat Genet. 1995;10(3):288-293.

53. Osawa Y, et al. Role of acid sphingomyelinase of Kupffer cells in cholestatic liver injury in mice. Hepatology. 2010;51(1):237-245.

54. Pettus BJ, et al. The sphingosine kinase 1 /sphingosine-1-phosphate pathway mediates COX-2 induction and PGE2 production in response to TNF- $\alpha$. FASEB J. 2003;17(11):1411-1421. 